\title{
Predicting results of mycobacterial culture on sputum smear reversion after anti-tuberculous treatment: a case control study
}

\author{
Chin-Chung Shu', Jann-Tay Wang ${ }^{2}$, Chih-Hsin Lee ${ }^{3}$, Jann-Yuan Wang ${ }^{2 *}$, Li-Na Lee ${ }^{4}$, Chong-Jen Yu²
}

\begin{abstract}
Background: Little is currently known regarding sputum smear reversion (acid-fast smear becomes positive again after negative conversion) during anti-tuberculous treatment. This study aimed to evaluate its occurrence in patients with pulmonary tuberculosis (TB) and identify factors predicting results of mycobacterial culture for smearreversion of sputum samples.

Methods: The retrospective review was performed in a tertiary referral center and a local teaching hospital in Taiwan. From 2000 to 2007, patients with smear-positive culture-confirmed pulmonary TB experiencing smear reversion after 14 days of anti-tuberculous treatment were identified.
\end{abstract}

Results: The 739 patients with smear-positive pulmonary TB had 74 (10\%) episodes of sputum smear reversion that grew Mycobacterium tuberculosis in 22 (30\%) (Mtb group). The remaining 52 episodes of culture-negative sputum samples were classified as the non-Mtb group. The anti-tuberculous regimen was modified after confirming smear reversion in 15 (20\%). Fourteen episodes in the Mtb group and 15 in the non-Mtb group occurred during hospitalization. All were admitted to the negative-pressure rooms at the time of smear reversion. Statistical analysis showed that any TB drug resistance, smear reversion within the first two months of treatment or before culture conversion, and the absence of radiographic improvement before smear reversion were associated with the Mtb group. None of the smear reversion was due to viable M. tuberculosis if none of the four factors were present.

Conclusions: Sputum smear reversion develops in 10\% of patients with smear-positive pulmonary TB, with 30\% due to viable M. tuberculosis bacilli. Isolation and regimen modification may not be necessary for all drugsusceptible patients who already have radiographic improvement and develop smear reversion after two months of treatment or after sputum culture conversion.

\section{Background}

Tuberculosis (TB) remains a global health problem despite near eradication in some developed countries [1-4]. In 2005, the incidence was 76 per 100,000 population in Taiwan, 80 per 100,000 in the Republic of Korea, and 600 per 100000 in South Africa [1,2]. To prevent further dissemination of Mycobacterium tuberculosis from TB patients, adequate anti-tuberculous treatment with the implementation of the Directly Observed Therapy (DOT) is important [5]. This is meant to achieve

\footnotetext{
* Correspondence: jywang@ntu.edu.tw

${ }^{2}$ Department of Internal Medicine, National Taiwan University Hospital, Taipei, Taiwan
}

negative conversion of sputum smear for acid-fast bacilli (AFB) and culture for M. tuberculosis.

In some TB patients, follow-up sputum smears occasionally reveal AFB after negative conversion (smear reversion). To avoid transmission, those with sputum smear reversion are usually admitted in a negative-pressure isolation room if hospitalization is indicated. The anti-tuberculous regimen is modified under the impression of treatment failure, drug resistance, or poor adherence. Indeed, only those with viable $M$. tuberculosis bacilli should receive proper isolation and further work-up. However, mycobacterial culture results are available only after 1-2 weeks using fluorometric culture technique, and 4-6 weeks by conventional solid culture medium [6]. The 
commercialized nucleic acid amplification tests, despite good sensitivity and specificity, are expensive and do not discriminate between viable and dead bacilli [7].

Therefore, from a practical standpoint, it is very important to understand how to differentiate among viable $M$. tuberculosis, dead bacilli, and non-tuberculous mycobacteria (NTM) in order to identify cases that remain infectious and reduce unnecessary expenditure of medical resources. This issue is not properly addressed in current literature. This retrospective study aimed to identify TB patients with smear reversion during anti-tuberculous treatment and to compare the clinical characteristics between those whose sputum samples remained culture-positive for $M$. tuberculosis and those whose sputum samples were culture-negative. The study also tried to identify factors predicting the results of mycobacterial culture upon sputum smear reversion after anti-tuberculosis treatment.

\section{Methods}

\section{Study subjects}

This study was conducted in a 2000-bed tertiary-care referral center in northern Taiwan and its branch, a 500-bed local teaching hospital in southern Taiwan. The Institutional Review Board of the hospital's Research Ethics Committee approved the protocol. By review of the database of mycobacterial laboratories, all patients with smear-positive, culture-confirmed pulmonary TB from 2000 to 2007 in the tertiary-care referral center and from 2004 to 2007 in the local teaching hospital were identified.

Patients whose sputum samples became smear-positive for AFB after having three consecutive smear-negative sputum samples and having received antituberculous treatment for more than 14 days (sputum smear reversion) were identified. They were classified into the M. tuberculosis group (Mtb group) if their sputum samples at smear reversion were culture-positive for M. tuberculosis, and the non-Mtb group if their sputum samples at smear reversion were culture-negative.

\section{Mycobacterial study}

Sputum samples were processed and pre-treated as previously described [8]. AFB smears of the processed samples were stained by the Kinyoun method and examined using standard procedures [9]. Smear grading was noted according to the American Thoracic Society guidelines [10]. If several sputum samples collected in the same period from a patient were smear-positive but with different grades, the highest was recorded as the patient's smear grading. From November 2006, the auramine-rhodamine fluorochrome method was used for screening before the smear was confirmed by the Kinyoun method [9].
The medium for primary mycobacterial isolation was Middlebrook 7H11 selective agar with antimicrobials (Remel Inc., Lexena, Kans.) and the fluorometric BACTEC technique (BACTEC Mycobacterium Growth Indicator Tube [MGIT] 960 system, Becton-Dickinson). Mycobacterial species were identified using conventional biochemical testing [9]. The susceptibility test of firstline anti-tuberculous drugs was examined using two concentrations of isoniazid $(0.2$ and $1.0 \mu \mathrm{g} / \mathrm{ml})$ and ethambutol $(7.5$ and $15 \mu \mathrm{g} / \mathrm{ml})$, and one concentration of rifampicin $(1.0 \mu \mathrm{g} / \mathrm{ml})$ [11]. For isoniazid and ethambutol, low-level resistance was defined as resistance.

\section{Terminology definition and data collection}

The patients' medical charts and interview records from TB case managers were reviewed to collect clinical data, including age, gender, underlying co-morbidity, symptoms, and results of laboratory tests and radiographic findings upon initial diagnosis and on sputum smear reversion. Management with smear reversion and outcome at the end of follow-up were also recorded.

In the two hospitals, patients with smear-positive pulmonary TB were requested to submit three sputum samples for acid-fast smear and mycobacterial culture every two weeks until smear conversion, and then monthly until culture conversion. Sputum culture conversion after anti-tuberculous treatment was achieved if three consecutive sputum samples were culture-negative for M. tuberculosis. The sputum mycobacterial load during smear reversion was considered "high" if there were multiple $3+$ or $4+$ positive smears, "medium" if there were multiple $2+$ positive smears, and "low" for the rest.

A single pulmonologist and a single radiologist both blinded to the clinical data interpreted the findings on chest radiography. If their opinions differed, the films were reviewed by another senior pulmonologist blinded to the results. Radiographically, "no improvement" was considered if the lesions seen on smear reversion did not decrease in size as compared to those before antituberculous treatment, or if there were new lesions.

Standard anti-tuberculous treatment was defined as daily isoniazid, rifampicin, ethambutol, and pyrazinamide (HREZ) for the first 2 months (intensive phase), followed by daily isoniazid and rifampicin for 4 months (continuation phase) [12]. Primary-care physicians modified the regimen based on any concomitant hepatic/ renal disease, adverse events, or results of susceptibility testing. The government began the Directly Observed Therapy-short course (DOTs), which covered the entire course of anti-tuberculous treatment, in April 2006. Treatment failure was defined as the presence of culturable $M$. tuberculosis in sputum samples after 4 months of anti-tuberculous treatment [13]. 


\section{Statistical analyses}

Inter-group differences were compared using the chisquare test or Fisher exact test for categorical variables, if appropriate. Time to smear reversion and survival were compared by the Kaplan-Meier method using the $\log$-rank test. Variables with a significant difference $(p<$ $0.05)$ in univariate analysis were further tested by multivariate logistic regression analysis to identify independent factors predictive of the results of mycobacterial culture on sputum smear reversion.

\section{Results}

Seven hundred and thirty-nine (739) patients with smear-positive culture-confirmed pulmonary $\mathrm{TB}$ were identified. The pre-treatment $M$. tuberculosis isolate was multi-drug resistant (MDR) in 49, resistant but not MDR in 114, and all susceptible in the remaining 576. There were 68 patients with 74 (10.0\%) episodes of sputum smear reversion, one with three episodes, and another four with two episodes. Mycobacterial culture of sputum samples on smear reversion yielded $M$. tuberculosis in 22 episodes, NTM in 5, and negative in the remaining 47 . The former $22(30 \%)$ was defined as the Mtb group and the rest as the non-Mtb group. The median number of sputum samples collected upon smear reversion was 3 (range: $2-6$ sets). Of the 46 episodes of smear reversion that developed after November 2006, nine (20\%) were due to viable $M$. tuberculosis bacilli. In the Mtb group, the pre-treatment M. tuberculosis isolate was MDR in five $(10 \%$ of the 49 patients with MDR TB) and was resistant but not MDR in six (5.3\% of the 114 patients with resistant but not MDR TB).
The clinical characteristics of the two groups were similar but more patients in the Mtb group had smear reversion within the first two months of anti-tuberculous treatment and before sputum culture conversion. More patients in the Mtb group also had no improvement in symptoms before smear reversion and were not treated under DOTs (Table 1). Four in the Mtb group developed smear reversion after nine months of antituberculous treatment. About half of the patients in each group had underlying diseases, which were most commonly diabetes mellitus and malignancy (Additional file 1: Table S1). Two patients in the non-Mtb group were co-infected by the human immunodeficiency virus (HIV). The Mtb group had a higher rate of resistance against tested first-line drugs and higher mycobacterial load (medium to high) on smear reversion (Table 2). A significantly higher proportion of patients in the Mtb group had no radiographic improvement before smear reversion than those in the non-Mtb group $(p=0.005)$.

Both groups were divided into two sub-groups each based on the susceptibility results of the pre-treatment M. tuberculosis isolates, and were compared (Additional file 1: Tables S1 and S2). The findings were similar except for: 1) the proportions of patients treated under DOTs were not significantly different $(p=0.073) ; 2)$ the proportions of patients whose anti-tuberculous regimen had ever been modified were significantly different $(p<$ 0.001 ); and 3 ) the time to smear reversion was significantly different (Figure 1).

The anti-tuberculous regimen was modified in 15 (20\%) episodes after knowing smear reversion. In the 29 episodes that occurred during hospitalization, including $14(64 \%)$ in the Mtb group and $15(29 \%)$ in the

Table 1 Clinical characteristics of patients with smear reversion

\begin{tabular}{lccc}
\hline & $\begin{array}{c}\text { Mtb group } \\
(\mathbf{n}=\mathbf{2 2})\end{array}$ & $\begin{array}{c}\text { Non-Mtb group } \\
(\mathbf{n}=\mathbf{5 2})\end{array}$ & $\boldsymbol{p}$ value \\
\hline Age $\geq 65$ years & $9(41 \%)$ & $24(46 \%)$ & 0.678 \\
Male gender & $16(73 \%)$ & $43(83 \%)$ & 0.330 \\
*Underlying co-morbid condition & $10(48 \%)$ & $30(57 \%)$ & 0.334 \\
Timing of smear reversion after treatment & & & \\
$\quad$ Median (range): days & $68(16 \sim 647)$ & $5(43 \sim 760)$ & $0.240^{\dagger}$ \\
$\quad$ Within 2 months & $9(41 \%)$ & $11(20 \%)$ & 0.002 \\
$\quad$ Before sputum culture conversion & $17(77 \%)$ & $15(29 \%)$ & 0.001 \\
*Symptoms not improved at smear reversion & $13(59 \%)$ & $19(37 \%)$ & 0.014 \\
Regimen modification before smear reversion & $13(59 \%)$ & $41(79 \%)$ & 0.073 \\
Receiving direct observed therapy & $9(41 \%)$ & $8(15 \%)$ & 0.118 \\
Mortality at the end of follow-up & $7(32 \%)$ & 11.1 & $0.178^{\dagger}$ \\
Median survival: months & 12.8 & $5(10 \%)$ & 0.051 \\
Treatment failure & $6(27 \%)$ & & \\
\hline
\end{tabular}

Data are no. (\%), unless otherwise indicated.

*The detail of underlying co-morbid condition and symptoms were described in additional file 1: Table S1.

${ }^{\dagger} p$ value was calculated by the Kaplan-Meier method. 
Table 2 Laboratory and radiographic findings in patients with smear reversion

\begin{tabular}{|c|c|c|c|}
\hline & $\begin{array}{c}\text { Mtb } \\
\text { group } \\
(\mathrm{n}=22)\end{array}$ & $\begin{array}{l}\text { Non-Mtb } \\
\text { group } \\
(\mathrm{n}=52)\end{array}$ & $\begin{array}{c}p \\
\text { value }\end{array}$ \\
\hline $\begin{array}{l}\text { Mycobacterial load at } \\
\text { smear reversion }\end{array}$ & & & 0.007 \\
\hline High & $3(14 \%)$ & $1(2 \%)$ & \\
\hline Medium & $8(36 \%)$ & $9(17 \%)$ & \\
\hline \multicolumn{4}{|l|}{$\begin{array}{l}\text { *Resistance of pre-treatment } \\
\text { Mtb isolate }\end{array}$} \\
\hline Any TB drug resistance & $11(50 \%)$ & $9(17 \%)$ & 0.004 \\
\hline Multidrug resistance & $5(23 \%)$ & $1(2 \%)$ & 0.009 \\
\hline \multicolumn{4}{|l|}{ Finding of chest film } \\
\hline Bilateral involvement at initial & $16(73 \%)$ & $33(63 \%)$ & 0.441 \\
\hline Cavitary lesion at initial & 7 (32\%) & $12(23 \%)$ & 0.431 \\
\hline $\begin{array}{l}\text { Not improved at smear } \\
\text { reversion }\end{array}$ & 19 (86\%) & $27(52 \%)$ & 0.005 \\
\hline \multicolumn{4}{|l|}{ Blood tests at reversion } \\
\hline $\begin{array}{l}\text { Leukocyte } \\
\text { (>11000 or }<4000 / \mu \mathrm{L})\end{array}$ & $6(27 \%)$ & $11(21 \%)$ & 0.555 \\
\hline Hemoglobin $(<12$ g/dL) & $13(63 \%)$ & $19(42 \%)$ & 0.055 \\
\hline Albumin $(<3.5 \mathrm{~g} / \mathrm{dL})$ & $4(36 \%)$ & $7(50 \%)$ & 0.495 \\
\hline Total Bilirubin (> $1.2 \mathrm{mg} / \mathrm{dL}$ ) & $2(11 \%)$ & $6(16 \%)$ & 0.614 \\
\hline Creatinine (> $1.5 \mathrm{mg} / \mathrm{dL}$ ) & $3(17 \%)$ & $4(9 \%)$ & 0.358 \\
\hline
\end{tabular}

Abbreviation: Mtb, Mycobacterium tuberculosis; TB, tuberculosis Data are no. (\%) or mean [no. of tested patients], unless otherwise indicated. *The details of resistance of pre-treatment isolate were described in additional file 1: Table $\mathrm{S} 2$.

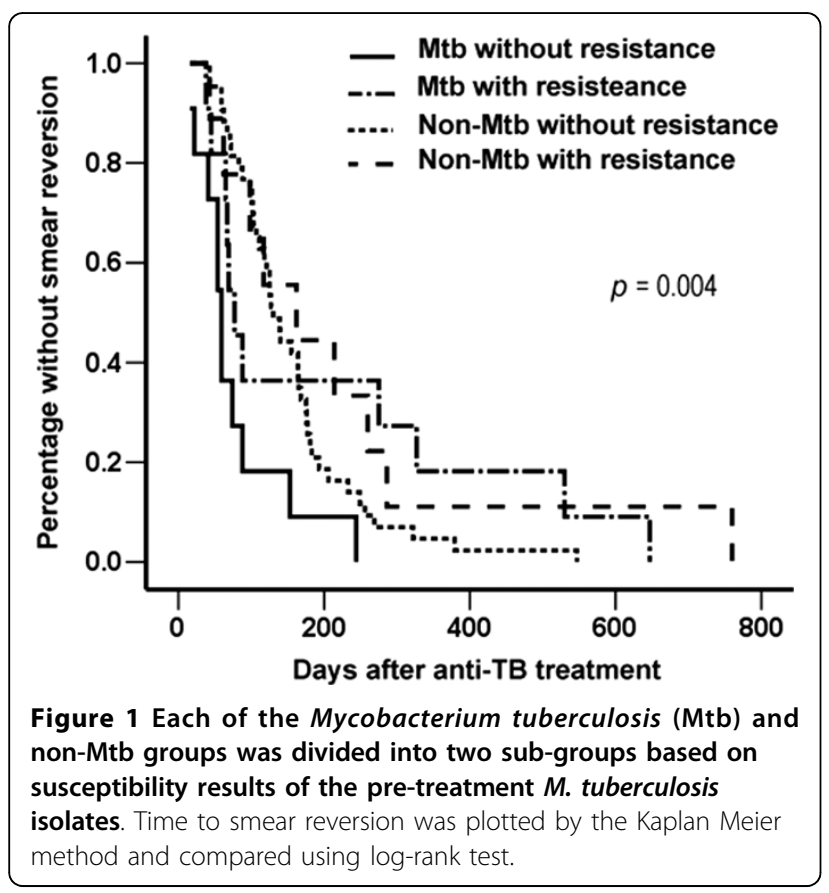

non-Mtb group, all were isolated in a negative-pressure room on smear reversion. The survival and outcome were similar in the two groups, though the Mtb group had a slightly, but insignificantly, higher rate of treatment failure than the non-Mtb group $(p=0.051$, Table 1).

Of the 22 episodes of M. tuberculosis-associated smear reversion (Additional file 1: Table S3 and S4), the antituberculous regimen was modified or interrupted before smear reversion in 13 (59\%). The most common causes were adverse events (seven episodes) and resistance of pre-treatment $M$. tuberculosis isolates (seven episodes). Anti-tuberculous treatment was interrupted due to ileus in one patient and poor adherence in another. In the nine episodes without treatment modification before smear reversion, four had underlying diseases while the other five occurred within the first two months of treatment. None of the patients with M. tuberculosis-associated smear reversion that occurred within two months of anti-tuberculous treatment had treatment failure.

Of the 22 clinical isolates of $M$. tuberculosis during smear reversion, drug susceptibility testing was repeated for 14, which revealed additional drug resistance in two isolates. Two isolates on smear reversion were multidrug resistant strains. The pre-treatment isolates of the two patients were all-susceptible in one and rifampicinresistant in the other.

Seven variables were included in the multivariate logistic regression analysis: treatment under DOTs strategy, presence of any TB drug resistance of the pre-treatment $M$. tuberculosis isolate, timing of smear reversion after treatment, smear reversion before sputum culture conversion, sputum mycobacterial load at reversion, and improvement of symptoms and radiographic findings before smear reversion (Additional file 2: Table S1). The results showed that factors independently predicting the results of mycobacterial culture on smear reversion were any TB drug resistance (OR: 10.33; 95\% C.I.:1.47-72.60), sputum smear reversion within 2 months after anti-tuberculous treatment (OR: 16.31; 95\% C.I.: 2.02-131.64), smear reversion before sputum culture conversion (OR: 29.55; 95\% C.I.: 4.07-214.72), and no radiographic improvement before smear reversion (OR: 23.57; 95\% C.I.: 2.49-223.38) (Table 3).

In order to evaluate the impact of treatment under DOTS, this variable was forcedly included in the final model of the multivariate analysis. The results showed that DOTS was not an independent predictor of culture results $(p=0.435)$ and the ORs for the four other variables did not significantly change.

The predictive values of the four associated factors were calculated (Table 4). If three or more factors existed, the 
Table 3 Factors associated with the presence of viable $M$. tuberculosis bacilli on sputum smear reversion in multivariate analysis

\begin{tabular}{lcccc}
\hline Characteristics & & Episodes no. & Cul (+) for Mtb & OR (95\% Cl.) \\
\hline Any TB drug resistance & Yes & 20 & $11(55 \%)$ & $10.33(1.47-72.60)$ \\
Mycobacterial load at reversion & No & 54 & $11(20 \%)$ & $11(52 \%)$ \\
Timing of Sm reversion after treatment & $\leq 2$ months & 21 & $11(21 \%)$ & $16.74(0.95-296.48)$ \\
& $>2$ months & 63 & $9(64 \%)$ & $16.31(2.02-131.64)$ \\
Sm reversion before Cul conversion & Yes & 28 & $13(22 \%)$ & \\
& No & 46 & $17(61 \%)$ & $29.55(4.07-214.72)$ \\
Findings of chest film at Sm reversion & Not improved & 46 & $19(41 \%)$ & $23.57(2.49-223.38)$ \\
\end{tabular}

Abbreviation: Cul, culture; Mtb, Mycobacterium tuberculosis; Sm, smear; TB, tuberculosis

The details of multivariate analysis were listed in Additional file 2: Table S1.

Table 4 Predicting the result of mycobacterial culture on smear reversion by four clinical factors

\begin{tabular}{ccccccc}
\hline Conditions & $\begin{array}{c}\text { Episodes of Sm } \\
\text { reversion }\end{array}$ & $\begin{array}{c}\text { Cul (+) } \\
\text { for Mtb }\end{array}$ & Se & Sp & PPV & NPV \\
\hline All & 1 & 1 & $5 \%$ & $100 \%$ & $100 \%$ & $72 \%$ \\
Any three & 1 & 1 & $5 \%$ & $100 \%$ & $100 \%$ & $72 \%$ \\
Any two & 33 & 21 & $95 \%$ & $78 \%$ & $64 \%$ & $98 \%$ \\
Anyone & 61 & 22 & $100 \%$ & $28 \%$ & $36 \%$ & $100 \%$ \\
\hline
\end{tabular}

Abbreviation: Cul, culture; Mtb, Mycobacterium tuberculosis; NPV, negative predictive value; PPV, positive predictive value; Se, sensitivity; Sm, smear; Sp, specificity

The four clinical factors include: 1) any one-drug resistance; 2) smear reversion within 2 months after treatment; 3) smear reversion before culture conversion and 4) no radiographic improvement before smear reversion

positive predictive value was $100 \%$. If none of them was present, the negative predictive value was $100 \%$. The positive and negative predictive values when any two factors were considered were $64 \%$ and $98 \%$, respectively.

\section{Discussion}

Sputum smear reversion after smear negative conversion during anti-tuberculous therapy is usually considered a sign of sub-optimal treatment or poor adherence $[14,15]$. Aside from evaluating and fostering patient compliance, new drugs are frequently added under the suspicion of emerging drug resistance. In addition, isolation in a negative-pressure room is deemed necessary if sputum smear reversion occurs during hospitalization. The results of this study suggest that sputum smear reversion develops in about $10 \%$ of patients with smear-positive, culture-confirmed pulmonary TB in an endemic area. Of these, $30 \%$ is caused by viable $M$. tuberculosis, which imply that altering treatment plans or doing contact isolation may not be necessary in the majority of those with sputum smear reversion. Therefore, understanding the factors predictive of results of mycobacterial culture upon sputum smear reversion is extremely important to reduce unnecessary medical cost.
In this study, factors independently associated with the presence of viable $M$. tuberculosis on sputum smear reversion include smear reversion within the first two months of anti-tuberculous treatment and before sputum culture conversion, the presence of drug resistance, and the absence of radiographic improvement before smear reversion. The results show that the presence of viable $M$. tuberculosis bacilli is less likely if none or one of the four factors is present. Hence, medical resources can be saved by applying the four factors to predict the results of mycobacterial culture.

The statistical analysis reveals that the absence of any radiographic improvement before sputum smear conversion is the strongest predictor for the results of mycobacterial culture. Although chest radiography is usually feasible and results can be available within the same day, the interpretation can sometimes be confounded during anti-tuberculous treatment. NTM pulmonary infection or colonization can result in higher smear grading and possibly, radiographic progression indistinguishable from pulmonary TB [16-18]. In addition, concomitant pulmonary malignancy or chronic lung disease, or paradoxical deterioration under successful treatment, renders the interpretation more difficult [19]. These factors also seriously compromise accuracy in evaluating subjective symptoms $[18,20]$. Nonetheless, the findings here suggest that careful interpretation and differentiation of etiologies responsible for the radiographic changes are important in managing TB patients with smear reversion.

Patients who develop M. tuberculosis-associated sputum smear reversion have higher any TB drug resistance rate $(50 \%)$ than those whose sputum samples are culturenegative for $M$. tuberculosis on smear reversion (17\%) and in the general TB population (17.7-20.4\%) [11,21]. Current evidence suggests that in areas with low prevalence of drug resistance, prescription modification is not necessary even if the pre-treatment isolate is mono-drug 
resistant, as long as there is improvement after one month of standard treatment [22]. However, patients in the Mtb group with drug resistance had smear reversal more than 2 months so that periodic sputum examinations and imaging studies in patients with drug-resistant TB may be needed for the early detection of emerging resistance and possible treatment failure.

This study reveals that the timing of sputum smear reversion is also an important predictor for the presence of viable M. tuberculosis bacilli. The underlying pathophysiology is most likely the rapidly decreased viability under effective combined anti-tuberculous chemotherapy. A sub-optimal, or even failed, regimen is not an important practical concern because none of those with sputum smear reversion within two months of antituberculous treatment has treatment failure. Consistent with previous studies, only smear positivity after two months of anti-tuberculous treatment is associated with poor treatment outcome [23,24].

Because sputum production is an irregular process, M. tuberculosis bacilli are potentially expectorated intermittently. Quantification of mycobacterial load at sputum reversion is determined by smear grading and reproducibility. However, a medium-to-high mycobacterial load on reversion reveals an insignificant association with the Mtb group by multivariate analysis. This is probably due to the presence of dead M. tuberculosis bacilli after anti-tuberculosis treatment. Another possible explanation may be the small sample size of the study, which limits the statistical power to detect true differences between the two groups. Underlying co-morbidity, even HIV infection, is not associated with culture-positive smear reversion under standard antituberculous treatment. As shown in previous studies, HIV infection is not associated with significantly longer smear conversion time and worse prognosis $[25,26]$.

This study has several limitations. First, although TB treatment guidelines are well established and implemented in clinical practice, the anti-tuberculous regimens and re-challenge protocols used in this study are heterogeneous due to the retrospective design. Second, the prevalence of smear reversion is likely to be underestimated because not all TB patients treated in the hospital during the study period regularly underwent sputum examinations. Third, due to the small sample size, conclusive generalizations cannot be made and other independent predictors, like drug adherence, for the presence of viable $M$. tuberculosis bacilli may not be statistically significant. Prospective, large-scale studies are warranted.

\section{Conclusions}

Sputum smear reversion develops in $10 \%$ of patients with smear-positive, culture-confirmed pulmonary TB in an endemic area. Of these, about $30 \%$ are due to viable M. tuberculosis bacilli. Proper contact isolation and investigation of possible sub-optimal treatment should be prioritised in those with drug-resistant $\mathrm{TB}$, those who develop smear reversion within two months after anti-tuberculous treatment or before sputum culture conversion, or those without radiographic improvement before smear reversion.

\section{Additional file 1: Clinical characteristics, laboratory and \\ radiographic findings in patients with smear reversion. Clinical characteristics, laboratory and radiographic findings of the patients with smear reversion were described, listed and compared according to the susceptibility of the patients' pre-treatment isolates. \\ Click here for file \\ [http://www.biomedcentral.com/content/supplementary/1471-2334-10- 48-S1.DOC] \\ Additional file 2: Factors associated with the presence of viable $M$. tuberculosis bacilli on sputum smear reversion. A table of multivariate analysis showed the details of the association between presence of viable $M$. tuberculosis bacilli on sputum smear reversion and seven significant factors from the univariate analysis. \\ Click here for file \\ [http://www.biomedcentral.com/content/supplementary/1471-2334-10- 48-S2.DOC ]}

\section{Acknowledgements}

We thank the members of the Taiwan Anti-Mycobacteria Investigation (TAMI) group for data collection and analysis. The members of the group include Jann-Yuan Wang; Li-Na Lee; Chong-Jen Yu; Pan-Chyr Yang; Hsin-Chih Lai; Wei-Juin Su; Chin-Chung Shu; Chih-Hsin Lee and Ming-Chih Yu. Funding source: This study was supported by the Institute for Biotechnology and Medicine Industry, Taiwan.

\section{Author details}

'Department of Traumatology, National Taiwan University Hospital, Taipei, Taiwan. ${ }^{2}$ Department of Internal Medicine, National Taiwan University Hospital, Taipei, Taiwan. ${ }^{3}$ Department of Internal Medicine, Buddhist Tzu Chi General Hospital, Taipei Branch, Taipei, Taiwan. ${ }^{4}$ Department of Laboratory Medicine, National Taiwan University Hospital, Taipei, Taiwan.

\section{Authors' contributions}

$J Y, L N$, and $C J$ participated in designing the study. CC participated in collecting all relevant data and wrote the manuscript. JT, $\mathrm{CH}, \mathrm{LN}$ and $\mathrm{CJ}$ were performed the data analysis. PC and JY conceived of the study, and participated in its coordination. All authors read and approved the final manuscript.

\section{Competing interests}

All of the authors declare no financial, professional, or otherwise personal interest of any nature or kind in related product, service, and/or company.

Received: 19 August 2009 Accepted: 6 March 2010

Published: 6 March 2010

\section{References}

1. Kuo SHS, Yi SW, Ting L, et al: Taiwan Tuberculosis Control Report 2007. Taipei: Centers for Disease Control, Department of Health, R.O.C (Taiwan), 42008.

2. Harling $G$, Ehrlich R, Myer $L$ : The social epidemiology of tuberculosis in South Africa: a multi-level analysis. Soc Sci Med 2008, 66(2):492-505.

3. Shimouchi A: Tuberculosis problems in the Asia-Pacific region. Respirology 2001, 6(1):75-78

4. Centers for Disease Control and Prevention (CDC): Trends in tuberculosisUnited States, 2008. MMWR Morb Mortal Wkly Rep 2009, 58(10):249-253. 
5. Albert RK, Iseman M, Sbarbaro JA, Stage A, Pierson DJ: Monitoring patients with tuberculosis for failure during and after treatment. Am Rev Respir Dis 1976, 114(6):1051-1060

6. Lu D, Heeren B, Dunne WM: Comparison of the Automated Mycobacteria Growth Indicator Tube System (BACTEC 960/MGIT) with LowensteinJensen medium for recovery of mycobacteria from clinical specimens. Am J Clin Pathol 2002, 118(4):542-545.

7. Wang JY, Lee LN, Hsu HL, Hsueh PR, Luh KT: Performance assessment of the DR. MTBC Screen assay and the BD ProbeTec ET system for direct detection of Mycobacterium tuberculosis in respiratory specimens. J Clin Microbiol 2006, 44(3):716-719.

8. Wang JY, Lee LN, Chou CS, et al: Performance Assessment of a NestedPCR Assay (the RAPID BAP-MTB) and the BD ProbeTec ET System for Detection of Mycobacterium tuberculosis in Clinical Specimens. J Clin Microbiol 2004, 42(10):4599-4603.

9. Pfyffer W, Christina Gutiérrez M, Brown-Elliott BA, Wallace RJ: Myobacterium. Manual of Clinical Microbiology Washington, DC: American Society for MicrobiologyPatrick R, Murray EJB, JH Jorgensen, MA Pfaller, Marie L Landry , 62007.

10. American Thoracic Society Centers for Disease Control and Prevention: Diagnostic Standards and Classification of Tuberculosis in Adults and Children. Am J Respir Crit Care Med 2000, 161(4 Pt 1):1376-1395.

11. Yu MC, Wu MH, Jou R: Extensively drug-resistant tuberculosis, Taiwan. Emerg Infect Dis 2008, 14(5):849-850.

12. Centers for Disease Control and Prevention (CDC): Treatment of tuberculosis. MMWR Recomm Rep 2003, 52(RR-11):1-77.

13. Luh KT, (ed): Taiwan guidelines for TB diangosis and treatment. Taipei: Center for Disease Control, Executive Yuan, Taiwan (R.O.C.), 32008.

14. Zhao FZ, Levy MH, Wen S: Sputum microscopy results at two and three months predict outcome of tuberculosis treatment. Int J Tuberc Lung Dis 1997, 1(6):570-572.

15. Salaniponi FM, Christensen JJ, Gausi F, Kwanjana JJ, Harries AD: Sputum smear status at two months and subsequent treatment outcome in new patients with smear-positive pulmonary tuberculosis. Int J Tuberc Lung Dis 1999, 3(11):1047-1048.

16. Huang CT, Tsai YJ, Shu CC, et al: Clinical significance of isolation of nontuberculous mycobacteria in pulmonary tuberculosis patients. Respir Med 2009 2009, 103(10):1484-91.

17. Griffith DE, Aksamit T, Brown-Elliott BA, et al: An official ATS/IDSA statement: diagnosis, treatment, and prevention of nontuberculous mycobacterial diseases. Am J Respir Crit Care Med 2007, 175(4):367-416.

18. Koh WJ, Yu CM, Suh GY, et al: Pulmonary TB and NTM lung disease: comparison of characteristics in patients with AFB smear-positive sputum. Int J Tuberc Lung Dis 2006, 10(9):1001-1007.

19. Wendel KA Alwood KS, Gachuhi $\mathrm{R}$, et al: Paradoxical worsening of tuberculosis in HIV-infected persons. Chest 2001, 120(1):193-197.

20. Shu CC, Lee $\mathrm{CH}$, Wang JY, et al: Non-tuberculous mycobacteria pulmonary infection in medical intensive care unit: the incidence, patient characteristics, and clinical significance. Intensive Care Med 2008, 34(12):2194-2201

21. Lai CC, Tan CK, Huang YT, et al: Extensively drug-resistant Mycobacterium tuberculosis during a trend of decreasing drug resistance from 2000 through 2006 at a Medical Center in Taiwan. Clin Infect Dis 2008, 47(7): e57-63.

22. Kim SJ: Drug-susceptibility testing in tuberculosis: methods and reliability of results. Eur Respir J 2005, 25(3):564-569.

23. Rieder HL: Sputum smear conversion during directly observed treatment for tuberculosis. Tuber Lung Dis 1996, 77(2):124-129.

24. Lienhardt $C$, Manneh $K$, Bouchier $V$, et al: Factors determining the outcome of treatment of adult smear-positive tuberculosis cases in The Gambia. Int J Tuberc Lung Dis 1998, 2(9):712-718.

25. Wang JY, Hsueh PR, Wang SK, et al: Disseminated tuberculosis: a 10-year experience in a medical center. Medicine 2007, 86(1):39-46.

26. Wang JY, Lee LN, Yu CJ, Chien YJ, Yang PC: Factors influencing time to smear conversion in patients with smear-positive pulmonary tuberculosis. Respirology 2009, 14(7):1012-1019.

\section{Pre-publication history}

The pre-publication history for this paper can be accessed here:

[http://www.biomedcentral.com/1471-2334/10/48/prepub] doi:10.1186/1471-2334-10-48

Cite this article as: Shu et al:: Predicting results of mycobacterial culture on sputum smear reversion after anti-tuberculous treatment: a case control study. BMC Infectious Diseases 2010 10:48.

\section{Submit your next manuscript to BioMed Central and take full advantage of:}

- Convenient online submission

- Thorough peer review

- No space constraints or color figure charges

- Immediate publication on acceptance

- Inclusion in PubMed, CAS, Scopus and Google Scholar

- Research which is freely available for redistribution
C Biomed Central 